MS21-03

\section{Polarizing multipolar atoms: a new toolbox for benchmarking and more}

Théo Leduc ${ }^{1}$, Emmanuel Aubert ${ }^{1}$, Christian Jelsch ${ }^{1}$, Cristian

Iordache $^{1}$, Enrique Espinosa ${ }^{1}$, Benoit Guillot ${ }^{1}$

1. CRM2, Université de Lorraine, Nancy, France

email: theo.leduc@univ-lorraine.fr

Ab initio calculations are becoming applicable on larger and larger molecules; nevertheless designing models of lower complexity is not devoid of interest for modeling biological complexes. Compared to point charge/moment models, electron density models add a rich layer of information: atomic basins, bond topology descriptors, etc. This study aims to improve electrostatic interaction energy calculations based on transferable multipolar atoms [1] with explicit consideration of dipole induction. We focus on polarizability calculations on isolated and interacting molecules taken from a high-quality theoretical benchmarking dataset, for which a detailed energy partition scheme is available [2]. Since polarizabilities change upon binding, transferability of this physical quantity between similar atoms is in question. To assess this problem, the interplay of theoretical polarizability variations with molecular geometry, external electric fields and interaction energies have been carefully examined. We will present our preliminary results along with the new tools we implemented in MoProSuite [3] for this study.

References:

[1] Domagała, S. et al. (2012). Acta Cryst., A68, 337-351.

[2] Bojarowski, S.A. et al. (2016). Chem. Phys. Chem, 17, 24552460.

[3] Guillot, B. (2014). Acta Cryst., A70, C279-C279.

Keywords: Polarizabilities, Dipole, Transferability
MS21-04

\section{The role of the crystal field to determine hydrogen atom positions in strong intramolecular hydrogen bonds}

Lorraine Andrade Malaspina ${ }^{1}$, Sajesh P. Thomas ${ }^{2}$, Kunihisa

Sugimoto $^{3}$, Simon Grabowsky ${ }^{1}$

1. Institute of Inorganic Chemistry and Crystallography, University of Bremen, Bremen, Germany

2. Department of Chemistry, Aarhus University, Aarhus, Denmark

3. Japan Synchrotron Radiation Research Institute (JASRI), Hyogo, Japan

email: lorraine.malaspina@gmail.com

The hydrogen maleate (HM) anion (Figure 1) has been investigated extensively in the past due to its very short intramolecular hydrogen bond. This hydrogen bond closes the HM anion to a seven-membered ring configuration in a resonance-assisted hydrogen bond (RAHB) fashion.[1]

There is a large number of crystal structures of hydrogen maleate salts in the Cambridge Structural Database (CSD) which show that the $\mathrm{O} \cdots \mathrm{O}$ distance varies from 2.361 to $2.540 \AA$ with a large variety of intermediate distances, with the hydrogen bond varying from perfectly symmetric to highly asymmetric. Neutron-diffraction experiments have shown that the proton position is highly dependent on the counter cation and hence on the intermolecular interaction pattern, exemplifying the flexibility of this compound regarding intermolecular interactions and crystalline environments.[1]

The crystalline environment polarizes the two oxygen atoms $\mathrm{O} 1$ and $\mathrm{O} 2$ to different extents. The bigger the difference in the polarization of the two oxygen atoms, the higher the asymmetry in the hydrogen atom position of the intramolecular RAHB. Consequently, hydrogen atom H1 shifts more closely to the oxygen atom that is less polarized by the environment. The asymmetry of the RAHB is therefore in direct relationship with the asymmetry of the intermolecular interaction pattern and the crystalline environment.

In this work the trajectory of the proton transfer is mapped through experimental electron density (ED) studies of a series of HM salts (cations: 4-aminopyridinium, 8-hydroxyquinolinium, $\mathrm{K}, \mathrm{Mg}, \mathrm{Ca}, \mathrm{Li}, \mathrm{Na}$ ) using high-resolution low-temperature synchrotron X-ray diffraction data measured at the beamline BL02B1 of SPring-8, Japan. The experimental electron density was modeled using the Hansen-Coppens multipole formalism,[2] and X-ray wavefunction refinement [3]. The response of the hydrogen atom position to the experimentally derived electric field imposed by the crystallographic environment will be presented and discussed. Changes in the character of the O-H...O bond will furthermore be characterized utilizing a complementary bonding analysis based on quantum crystallography. 


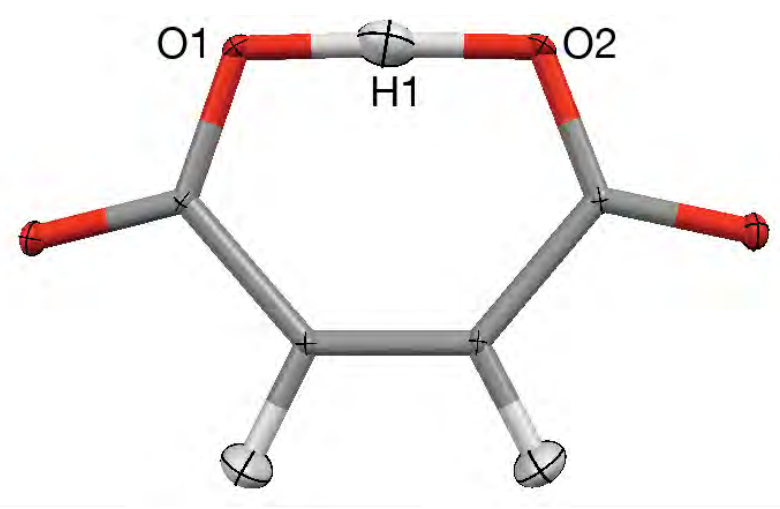

References:

[1] Malaspina, L. A. et al. (2017). Cryst. Growth Des. 17, 38123825 .

[2] Hansen, N. K. \& Coppens, P. (1978). Acta Cryst. A34, 909-921.

[3] (a) Jayatilaka, D. (1998). Phys. Rev. Lett. 80, 798-801.

(b) Jayatilaka, D. \& Grimwood, D. J. (2001). Acta Cryst. A57, 76-86.

(c) Jayatilaka, D. \& Grimwood, D. J. (2001). Acta Cryst. A57, 87-100.

Keywords: Experimental electron density, electric field, crystalline environment

\section{MS21-05}

\section{Quantum crystallography towards "quantitative crystal engineering"}

Sajesh P Thomas ${ }^{1}$, Peter R. Spackman ${ }^{2}$, Khidhir Alhameedi², Dylan Jayatilaka $^{2}$, Bo B. Iversen ${ }^{1}$, Mark A. Spackman ${ }^{2}$

1. Department of Chemistry, Aarhus 8000, Denmark

2. School of Molecular Sciences, The University of Western Australia, Perth, Australia

email: sajeshpthomas@gmail.com

Understanding solid-state supramolecular chemistry requires quantitative insights into the nature and strength of intermolecular interactions. Over the past few decades, charge density multipole modelling (CDMM) formalism has contributed significantly to the understanding of chemical bonding in crystals [1]. However, the applicability and accuracy of CDMM in the intermolecular regime is a grey area. This is owing to the issues related to the partitioning of electron density in the intermolecular space using CDMM. Recent developments in the high resolution X-ray quantum crystallography offer significant prospects in this context. I will discuss recent results on the studies of intermolecular interactions, their strength and nature in terms of bond orders, electron density and interaction energies derived from both experiment and theory. Especially the applications of such quantitative descriptors will be discussed in the context of crystal engineering of molecular solids, their relative stabilities and intriguing mechanical properties[2,3].

References:

[1] Stalke, D. (2011) Chem. Eur. J. 17, 9264.

[2] Turner M. J., Thomas S. P., Shi M. W., Jayatilaka D. and Spackman M. A., (2015), Chem.Commun, 51, 3735.

[3] Thomas, S. P., Shi, M. W. ,. Koutsantonis, G. A, Jayatilaka, D., Edwards, A. J., Spackman, M. A. (2017) Angew Chem Int Ed Engl., 129, 29, 8588-8592.

Keywords: charge density, crystal engineering, intermolecular interactions 